\title{
Definición de las competencias que debe adquirir el futuro médico. Formación de los profesores
}

\author{
J.M. Peinado-Herreros
}

\section{Introducción}

Una de las consecuencias más positivas del debate, que en su momento abrió el proceso de convergencia europea en España, fue la introducción del término 'competencia' y, en consecuencia, la necesidad de su evaluación con instrumentos fiables.

Por otra parte, y a fin de alcanzar de forma correcta uno de los objetivos principales de la reforma -la elaboración de nuevos planes de estudio basados en competencias-, se ha planteado como necesidad prioritaria la formación del profesorado, fundamentalmente en aspectos metodológicos, conceptuales, prácticos y evaluativos de la enseñanza.

\section{Antecedentes normativos}

La Directiva Europea 2005/36/CE del Parlamento Europeo y del Consejo relativa al reconocimiento de cualificaciones profesionales del 7 de septiembre de 2005 ha salvado los estudios de medicina del intenso proceso de desregulación. Ésta, al igual que en 1993, establece las necesidades de formación en las denominadas 'condiciones mínimas de formación (conocimientos y competencias) básicas del médico’.

Por otra parte, la Ley de Ordenación de las Profesiones Sanitarias, LOPS (2003) no hace referencia alguna a los contenidos del proceso formativo durante el grado y vuelve a manifestar el divorcio entre salud y educación. Las facultades de medicina no han evaluado tradicionalmente la formación clínica, y han prestado especial atención a los conocimientos enciclopédicos, como forma de garantizar el máximo rendimiento en la prueba de acceso a la formación especializada. La publicación de la LOPS fue acogida con expectación, ya que independientemente del proceso de Bolonia, introducía aspectos que de forma necesaria condicionarían la formación, tales como la evaluación de habilidades clínicas y de comunicación (LOPS, art. 22: 'El acceso a la formación sanitaria especializada se efectuará a través de una convocatoria anual que, en todo caso, consistirá en una prueba, o conjunto de pruebas, que evaluará conocimientos teóricos y prácticos y las habilidades clínicas y comunicativas, así como en una valoración de los méritos académicos y, en su caso, profesionales de los aspirantes. Asimismo, podrán establecerse pruebas específicas por especialidades troncales. Disposición transitoria primera: 'El nuevo modelo de prueba para el acceso a la formación sanitaria especializada previsto en el artículo 22.2 de esta ley se implantará de manera progresiva durante los ocho años posteriores a la entrada en vigor de esta norma'). Sin embargo, tras cinco años desde su publicación nada se ha realizado en el ministerio a fin de proceder a su 'progresiva implantación. Tampoco se han dado los pasos pertinentes con el fin de conectar el grado y la nueva estructura troncal de la formación especializada.

\section{El Libro Blanco de la titulación de medicina}

Esta obra constituye el primer documento, de carácter nacional, consensuado entre las facul-
Exdecano de la

Facultad de Medicina. Universidad de Granada. Ex presidente de la CND. Coordinador del proyecto ANECA para el diseño de planes de estudio.

E-mail jpeinado@ugr.es 


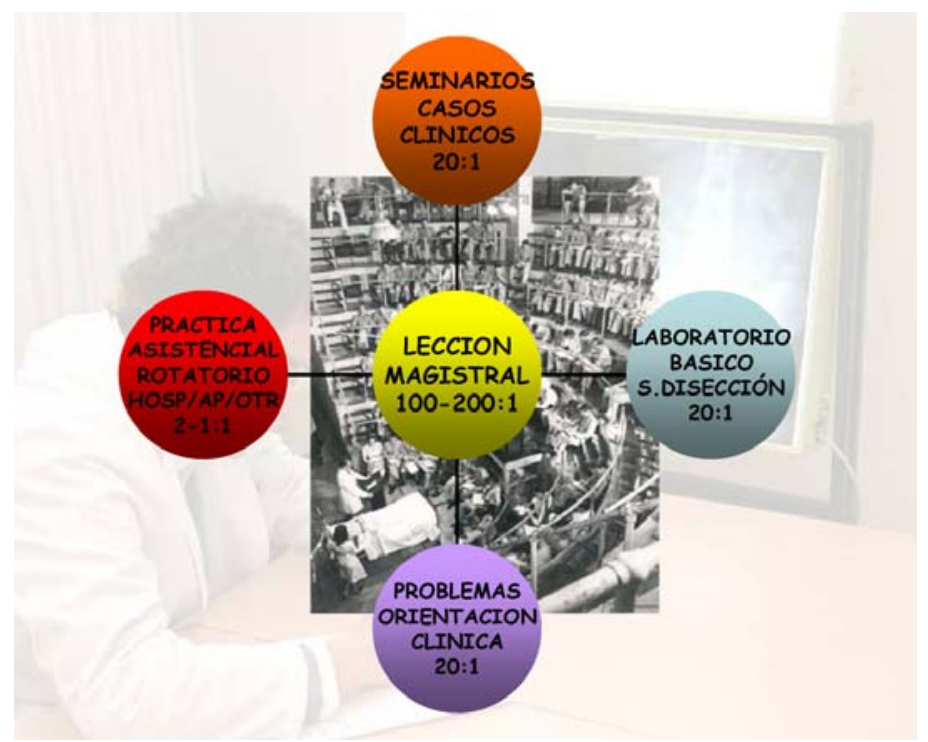

Figura 1. Diagrama en el que se muestra la configuración más frecuente del modelo formativo actual en las facultades de medicina españolas en torno a la lección magistral. Se especifican las ratios deseables en cada actividad alumno-profesor. (Los laboratorios de prácticas en disciplinas básicas -o preclínicas- como bioquímica, histología o microbiología y otras, junto a las salas de disección en anatomía, suelen estar organizados en relación con la docencia teórica. Recientemente, en algunas facultades, los departamentos básicos han introducido seminarios de problemas de orientación clínica, también basados en los contenidos teóricos de cada disciplina. En todas las facultades de medicina españolas existe formación clínica en hospitales y, en menor medida, en atención primaria u otros centros asistenciales. Los seminarios de casos clínicos no están generalizados.) .

tades de medicina españolas, en el que se definen las competencias genéricas y específicas que debería tener el graduado en medicina. Los antecedentes deben buscarse en el documento elaborado por el International Institute for Medical Education. En éste se basa la Orden Ministerial ECI/332/2008.

Aunque el último objetivo del Libro Blanco es su utilización como herramienta en la elaboración de planes de estudio, éste entra en el desarrollo pormenorizado de las competencias. Se organizan bloques y subbloques de materias que el Ministerio de Educación (Orden Ministerial, 2008) denominará años más tarde módulos y materias, que, a su vez, las facultades organizarán en asignaturas.

\section{Metodología docente}

Tradicionalmente, nuestro sistema formativo universitario se ha basado en la transmisión de conocimientos: la lección magistral. En torno a ésta se ha estructurado, en su caso, el resto de actividades formativas y el sistema de evaluación. La formación y evaluación de prácticas en materias básicas siempre ha estado presente en nuestros currículos, aunque su peso en la calificación ha sido muy desigual y en la mayoría escaso o nulo. Por otra parte, la formación clínica en hospitales universitarios ha sido en general una delimitación horaria, sin la determinación de objetivos, contenidos y mucho menos evaluación. El aprendizaje de habilidades clínicas ha quedado supeditado al interés del alumno y del profesor, y sus contenidos han dependido del azar. Sólo recientemente, en algunas facultades, se han comenzado a definir la estructura y los contenidos de la formación clínica y tímidamente su evaluación. Para ello ha sido necesario el montaje -no siempre posiblede laboratorios de habilidades clínicas, que son caros, difíciles de mantener y con requerimientos específicos de profesorado (Fig. 1).

A pesar de los tímidos cambios metodológicos introducidos en los últimos años, desde mi 


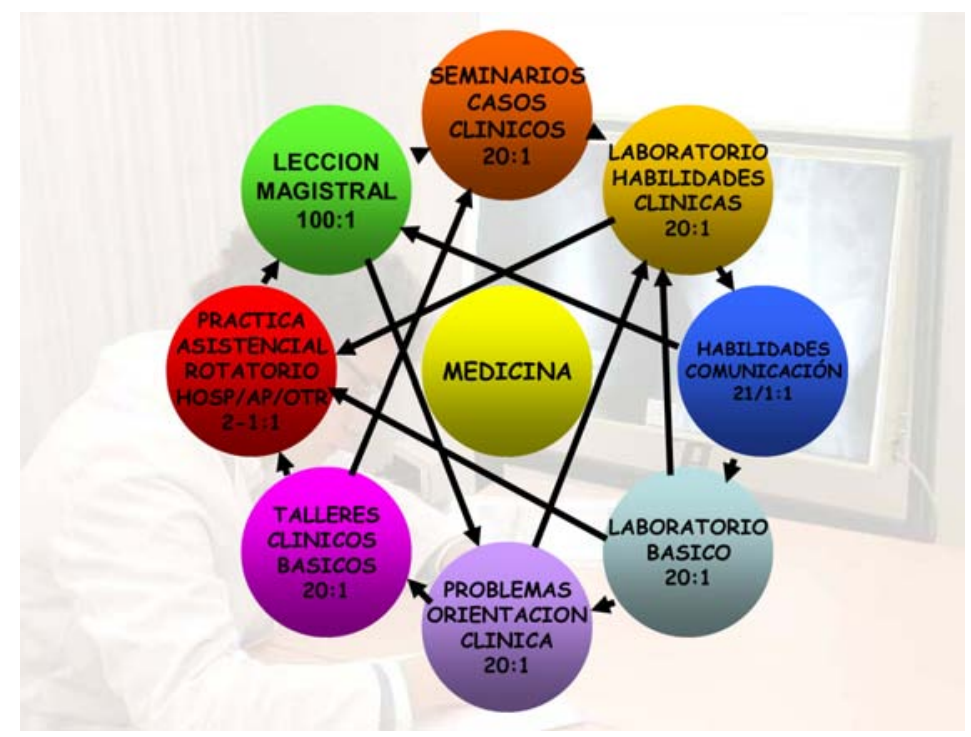

Figura 2. Representación de los diferentes componentes que podrían integrar el proceso formativo médico. Se especifican las ratios deseables en cada actividad alumno-profesor.

punto de vista, la enseñanza moderna de la medicina debe estructurarse siguiendo un nuevo modelo, en el que todos los elementos formativos son igualmente importantes. La lección magistral debe seguir jugando un papel, pero deben incorporarse otros procedimientos didácticos, tal como se muestra en la figura 2.

Los sistemas de evaluación también deben cambiar de forma sustancial. Es necesario evaluar los conocimientos, pero también las habilidades y las actitudes. Uno de los elementos recomendables a la hora de evaluar la formación clínica sería el denominado 'cuaderno del estudiante', una especie de 'portafolio' en el que se recogerían las actividades realizadas en el proceso de aprendizaje. Lógicamente, los objetivos tendrían que estar definidos previamente a modo de checklist, de manera que cada tutor pudiera evaluar de forma continuada. Además, y como evaluación final, el estudiante debería realizar una prueba tipo evaluación clínica objetiva y estructurada (ECOE).

Queda por definir la relación entre la evaluación antes mencionada y la prueba de acceso a la formación especializada, elemento que sin duda condicionará el proceso formativo anterior.

\section{Formación y evaluación del profesorado}

Definidas las competencias y el proceso de aprendizaje y evaluación, hay que dejar constancia de que cualquier sistema nuevo no tendrá éxito si no se dispone de un profesorado colaborador dispuesto a llevarlo a cabo. En este sentido, los condicionantes de la metodología docente, así como de los incentivos a los que se enfrente el profesorado para cambiar tradiciones fuertemente arraigadas será fundamental.

En general, los médicos dedicados a la formación enseñan sin ningún tipo de preparación previa. Éste sería otro de los objetivos deseable del proceso de Bolonia: garantizar una correcta formación de los enseñantes. Sin embargo, en nuestras universidades, tras un breve período de expectación, se ha vuelto a imponer la importancia de la investigación frente a la docencia, casi entendida como la acumulación de años y créditos, otorgados de forma automática (sólo muy tímidamente se han introducido evaluaciones periódicas de la actividad docente del profesorado con escasa repercusión en la promoción académica). 

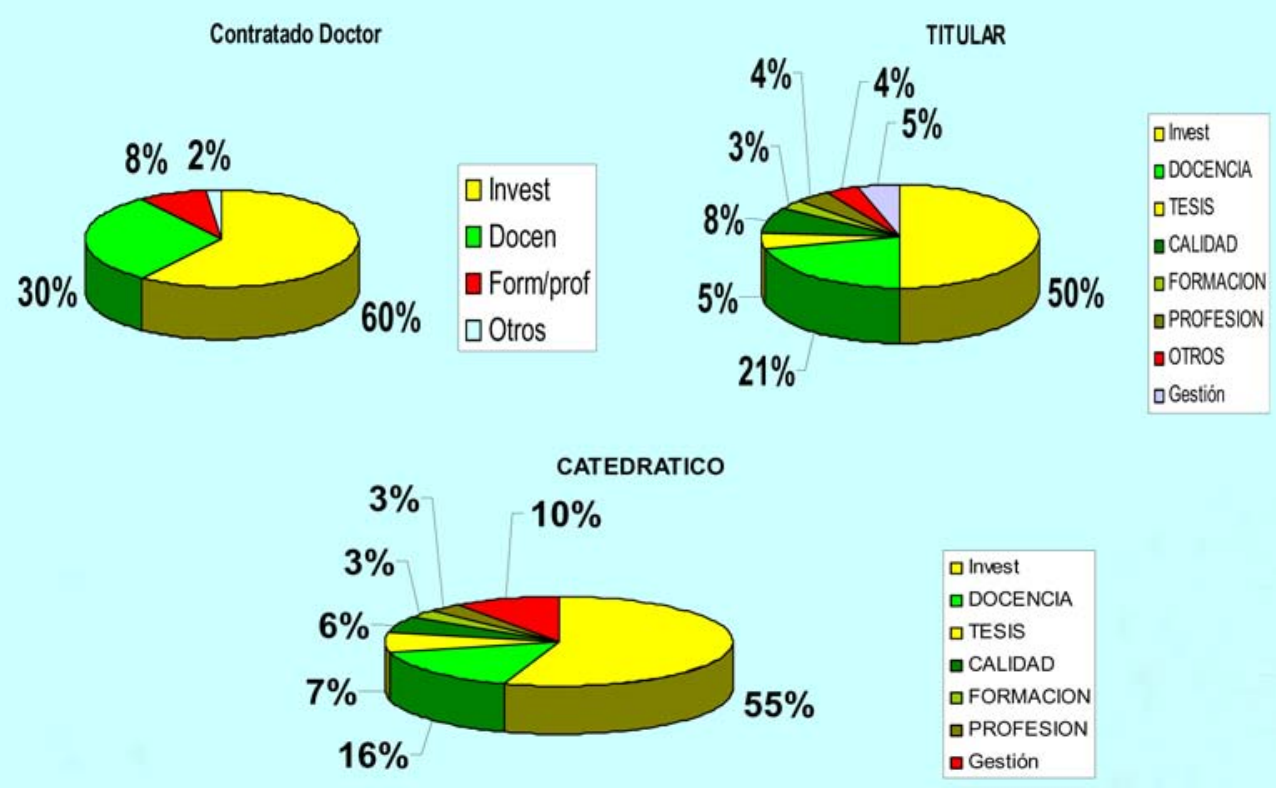

Figura 3. Peso porcentual de los componentes de investigación, docencia y otros, de acuerdo con la actual normativa y Programa Academia de la Agencia Nacional de Evaluación, Calidad y Acreditación (ANECA).

En 2001, la publicación de la Ley Orgánica de Universidades (LOU) introducía nuevas figuras de profesorado y nuevos sistemas de acceso a dichas figuras. Desgraciadamente, la falta de voluntad o simplemente el desconocimiento de los responsables de elaborar la norma desaprovechó una oportunidad excelente para abordar la problemática del profesorado médico, que se mantuvo como en la Ley de Reforma Universitaria (LRU). Solamente los profesores funcionarios (titular o catedrático) podían compatibilizar su actividad asistencial y docente a través de la figura del profesor vinculado. La modificación en 2007 de la LOU introdujo la posibilidad de vincular a la actividad asistencial a los profesores contratados doctores, al ser ésta una figura laboral con carácter permanente. Transcurrido más de un año desde su publicación, creo poder afirmar que no existe en España ningún profesor contratado doctor vinculado, al no haberse desarrollado la normativa propia de acceso a dicha figura. La figura del profesor asociado de ciencias de la salud no se ha modificado.

Un aspecto que se debe destacar, y de cierta trascendencia en la nueva carrera académica del profesorado médico, ha sido el sistema de acceso a las diferentes figuras de profesorado. El sistema de habilitación nacional, definido en la LOU de 2001, supuso un frenazo en la convocatoria de plazas por parte de las universidades, que fue mucho más acusada en el caso de plazas vinculadas. La puesta en marcha de los sistemas de acreditación de la nueva LOU ha reducido algunos inconvenientes, aunque han aparecido otros nuevos difíciles de superar. A mi juicio, el más destacado es la sobrevaloración de la actividad investigadora frente a otros méritos como los docentes, los profesionales u otros. La fuerte demanda asistencial en la que se ven inmersos los profesores vinculados, particularmente en las áreas quirúrgicas, ha mermado con carácter general las posibilidades de realizar investigación puntera. El porcentaje de profesores con evaluación positiva (uno o más) de sexenios por la Comisión Nacional Evaluadora de la Actividad Investigadora (CNEAI) en el período 1989-2005 en Cirugía (46\%), Ginecología (30\%), ORL (26\%), Psiquiatría (37\%) o Radiología (46\%), es sensiblemente superior al promedio nacional $(72 \%)$ o al obtenido por el profesorado en el área de 
ciencias de la salud (70\%). Por otra parte, el establecimiento en la nueva LOU de baremos para la acreditación, en los que prima sensiblemente la investigación, $60 \%$ para contratado doctor, $50 \%$ para profesor titular y $55 \%$ para catedrático, con una valoración de la actividad profesional entre un 3 y un $8 \%$, hará muy difícil la acreditación en determinadas áreas clínicas. Puede añadirse que la docencia, aunque sea de calidad, goza de escaso prestigio frente a la investigación en nuestro sistema universitario (Fig. 3).

Todo esto, junto a una muy escasa coordinación entre la recientemente regulada carrera profesional en el sector sanitario y la carrera académica, además de unas mejores expectativas de futuro, incentivos y reconocimiento social, fuera de la universidad, ha menguado considerablemente la posibilidad de la incorporación de jóvenes médicos con vocación universitaria y, en consecuencia, la formación de profesorado clínico. De mantenerse las actuales circunstancias, es previsible que en ciertas áreas, desaparezca de las facultades de medicina el profesorado funcionario en los próximos años.

$\mathrm{Si}$ corresponde a las instituciones universitarias asegurar la calidad de su profesorado mediante políticas de personal adecuadas y procesos de selección, formación, evaluación e incentivación pertinentes, no cabe duda de que hay mucho camino por recorrer en el ámbito de la formación del profesorado médico.

En este sentido se hace necesario crear programas específicos de formación del profesorado médico. En cualquier caso, el profesor, además de conocer su disciplina, debe poseer formación pedagógica y didáctica suficiente, que le haga hábil en su profesión de transmisor de saberes, que ponga al alcance de los alumnos las competencias, con la aplicación de métodos y tecnologías convenientes para que cumpla con eficiencia su misión de enseñar y de educar. A esto podría colaborar la creación en cada centro de unidades de educación médica, entre cuyas funciones se encontraría la formación del profesorado, tal como ya ocurre en algunas facultades de medicina.

Finalmente, se necesita recuperar el control del proceso enseñanza-aprendizaje en la titulación. Para esto es necesaria la coordinación de contenidos en las distintas materias, con un pa- pel creciente de los decanatos, a los que se debe dotar de capacidad ejecutiva en el control del desarrollo del programa docente o de las propias unidades de educación médica.

\section{Bibliografía}

1. Bologna Working Group on Qualifications Framework. Report on a framework for qualifications of the European Higher Education Area. December; 2004. Cap. 2.4.1, p. 18. Véase también 'Using Learning outcomes'. Edimburgh, July 1-2; 2004.

2. Directiva Europea 2005/36/CE del Parlamento Europeo y del Consejo relativa al reconocimiento de cualificaciones profesionales de 7 de septiembre de 2005.

3. Global Minimum Essential Requirements in Medical Education. Institute for International Medical Education Core Committee. Educ Med 2003; 6: 13-4.

4. Ley 44/2003, de 21 de noviembre, de ordenación de las profesiones sanitarias. BOE 280, de 22 de noviembre de 2003. p. 41442-58.

5. Peinado-Herreros JM. Libro Blanco. Título de Grado en Medicina. Madrid: ANECA; 2006. URL: http://www. aneca.es/activin/docs/libroblanco_medicina_def.pdf [15.11.2008].

6. Memoria sobre la situación del profesorado numerario con respecto a los sexenios de investigación. Comisión Nacional de Evaluación de la Actividad Investigadora. Año 2005. Ministerio de Ciencia e Innovación. URL: http://www.micinn.es/ciencia/cneai/files/2005-memoria-situacion-prof-sin-teu.pdf.

7. Miller GE. The assessment of clinical skills/competence/ performance. Acad Med 1992; 65 (Suppl): S63-7.

8. Orden ECI/332/2008, de 13 de febrero, por la que se establecen los requisitos para la verificación de los títulos universitarios oficiales que habiliten para el ejercicio de la profesión de Médico. BOE 40 de 15 de febrero de 2008. p. 8351-5.

9. Real Decreto $1393 / 2007$, de 29 de octubre, por el que se establece la ordenación de las enseñanzas universitarias oficiales. BOE 260 de 30 de octubre de 2007. p. 44037-48.

10. González J, Wagenaar R, eds. Tuning educational structures in Europe. Informe final. Fase Uno. Bilbao: Universidades de Deusto y Groningen; 2003.

11. Van der Vleuten C. Validity of final examinations in undergraduate medical training. Br Med J 2000; 11: 1217-9.

12. Wojtczak A. Glosario de términos de educación médica. Educ Med 2003; 6 (Supl 2): S21-56. 